\title{
Comparison of urinary and sexual patient-reported outcomes between open radical prostatectomy and robot-assisted radical prostatectomy: a propensity score matched, population-based study in Victoria
}

\author{
Michael Rechtman ${ }^{1}$, Andrew Forbes' ${ }^{1}$, Jeremy L. Millar ${ }^{1,2}$, Melanie Evans' ${ }^{1}$, Lachlan Dodds ${ }^{3,4}$,
}

Declan G. Murphy ${ }^{5,6}$ and Sue M. Evans ${ }^{1,7^{*}}$

\begin{abstract}
Background: Robot-assisted radical prostatectomy (RARP) rates have been increasing worldwide despite a lack of evidence of superior patient-reported outcomes (PROs) compared to open radical prostatectomy (ORP).

Methods: This retrospective study included men who contributed data to the Prostate Cancer Outcomes RegistryVictoria (PCOR-Vic), underwent ORP or RARP between January 2014 and May 2018, and completed the EPIC-26 questionnaire 12 months post-surgery. Urinary and sexual bother items, the urinary incontinence domain score, the urinary irritative/obstructive domain score, the sexual domain score and the pad usage item from the EPIC-26 questionnaire were compared between the two cohorts. Unmatched and propensity score matched cohorts were used to determine if there were differences in urinary and sexual PROs between ORP and RARP after accounting for the patient case-mix and surgeon characteristics.
\end{abstract}

Results: Of 3826 patients undergoing radical prostatectomy (RP), 1047 received ORP and 2779 received RARP. Propensity score matching reduced the magnitude of the observed differences in four out of six outcomes (urinary bother, urinary incontinence domain, pad usage and sexual domain). Using a propensity score matched cohort, there were no statistically significant differences for RARP patients, compared to ORP patients, in terms of urinary bother $(\mathrm{Rd}=0.47 \%, P=0.707)$, urinary incontinence domain scores (Coeff $=-0.84, P=0.506)$, urinary irritative/obstructive domain scores (Coeff $=1.03, P=0.105)$, pad usage $(R d=-0.75 \%, P=0.771)$ and sexual bother $(R d=-0.89 \%$, $P=0.731$ ). RARP patients had slightly higher sexual domain scores (Coeff $=3.65, P=0.005$ ).

Conclusion: There were no differences in urinary PROs between ORP and RARP when assessed 12 months postsurgery. The sexual domain slightly favoured RARP, however this was not deemed clinically significant.

Keywords: Radical prostatectomy (RP), Open radical prostatectomy (ORP), Robot-assisted radical prostatectomy (RARP), Patient-reported outcomes (PRO), Expanded prostate cancer index composite (EPIC) questionnaire

*Correspondence: sue.evans@cancervic.org.au

1 School of Public Health and Preventive Medicine, Monash University,

533 St Kilda Road, Melbourne, VIC 3004, Australia

Full list of author information is available at the end of the article

\section{Background}

Radical prostatectomy (RP) is the most commonly used treatment for patients with localised prostate cancer and life expectancy greater than 10 years [1]. Open 
radical prostatectomy (ORP) and robot-assisted radical prostatectomy (RARP) are the two main RP approaches performed in Victoria [2]. The increased availability of robotic surgery has led to the use of a robotic approach in the majority of RPs in Victoria [3].

The American Urological Association and the European Association of Urology guidelines for localised prostate cancer state that there is no evidence of different urinary or sexual functional outcomes between ORP and RARP, however the evidence level is rated low in both [4, 5]. The largest randomised controlled trial (RCT) comparing ORP and RARP found no difference in oncological and urinary and sexual functional outcomes at 12- and 24-months following surgery [6, 7]. However, minimally invasive surgeries such as RARP have gained popularity globally due to the potential for reduced morbidity [8]. This may be due to improved perioperative outcomes, such as decreased blood loss, improved short-term postoperative outcomes, such as reduced postoperative pain and length of hospital stay [6], and clinician and patient preference [8]. Marketing campaigns, hospital and urologist competition, and centralised health systems may have also contributed to the perception that RARP is superior $[9,10]$. Furthermore, patient and surgeon characteristics may differ significantly between ORP and RARP cohorts [11]. Due to conflicting evidence and its increasing adoption [12], ongoing comparison of outcomes is warranted that is focussed on issues that impact quality of life. For this reason, observational studies and prostate cancer clinical registries have grown in number [13]. Population-based clinical registries allow stronger inferences to be made about the population compared to single- or multi-centre studies due to volume-outcome relationships [14].

This paper used a population-based clinical registry to compare urinary and sexual PROs from the EPIC-26 questionnaire of men undergoing ORP and RARP at oneyear following surgery.

\section{Methods \\ PCOR-Vic}

Data for this study were obtained from the PCOR-Vic, which collects clinical and patient-reported data on men diagnosed with prostate cancer from contributing institutions in Victoria [15]. Recruitment and data collection methods of the PCOR-Vic have previously been described [15]. At approximately 12 months post-diagnosis for men on active surveillance or watchful waiting, and 12 months post initial active treatment, participants are invited to complete the EPIC-26 quality of life questionnaire [16] via telephone, email or paper form [15].

\section{Data collection}

PCOR-Vic provided a dataset with a range of demographic, diagnostic and follow-up variables. Socioeconomic status was calculated using the patient's postcode to determine the Australian Bureau of Statistics index of relative socio-economic advantage and disadvantage (IRSAD) for the 2016 year [17]. Year of attaining surgical specialisation was collected from the Australian Health Practitioner Regulation Agency website or directly from surgeons.

\section{Patients}

Patients were included if they attended a participating institution of the PCOR-Vic, did not opt out of the registry, received an ORP or RARP between January 2014 and May 2018, and completed at minimum the urinary and sexual bother items of the EPIC-26 questionnaire between 0.7 to 1.3 years following RP.

\section{Outcomes}

Urinary outcomes were the EPIC-26 urinary bother item (dichotomised into moderate/big bother, and small/very small/no bother, consistent with cut-off points reported elsewhere) [14, 18], urinary incontinence and urinary irritative/obstructive domain scores, and pad usage (dichotomised into 'no pads per day' and ' $\geq 1$ pad per day') [19]. Sexual outcomes were the EPIC-26 sexual bother item (dichotomised into moderate/big bother, and small/very small/no bother) and sexual domain scores [19].

\section{Statistical analysis}

Descriptive analyses included medians and interquartile ranges for continuous data. Associations were determined using independent $\mathrm{t}$-tests for continuous data and chi-squared tests for categorical data. A two-tailed 5\% significance level was used throughout.

Propensity score matching was used to assess differences in urinary and sexual outcomes between ORP and RARP after accounting for the differences in patient and surgeon characteristics. Detailed steps involved in propensity score matching are described elsewhere [20, 21]. The propensity score refers to the estimated probability of receiving one of the treatment options. It was defined as the probability of receiving RARP, as opposed to ORP. Propensity scores were calculated for each patient using a logistic regression model based on the preoperative factors of age at surgery, PSA at surgery, surgeon's years since specialisation, National Comprehensive Cancer Network (NCCN) risk category, hospital location (metropolitan vs. regional), hospital type (public vs. private), IRSAD quintile and year of surgery.

The propensity score matching estimator used was a nearest neighbour 1:1 matching model, with replacement 
and no calipers. Therefore each patient in both treatment groups was matched to a patient in the other treatment group based on propensity scores. This model was used to define similarity in order to find the closest matches across the population. The matching estimator imputed the missing potential outcome for each individual (i.e. the outcome if they had received the other treatment option). Each potential outcome became an observation in the data.

In this manner, two groups of patients were formed that were similar on their propensity scores. After matching, the covariates used in calculating the propensity scores were checked for balance across the two treatment groups using a maximum standardised difference of $10 \%$, as previously recommended [20,22].

The average treatment effect for each outcome was estimated before and after propensity score matching. These were expressed as risk differences (RARP minus ORP) for binary variables and as mean differences (RARP minus ORP) for continuous variables.

All analyses were conducted using STATA version 15.1, with $p$ values $\leq 0.05$ considered statistically significant. The matched analyses used the teffects command. Ethics approval was obtained from the Monash University Human Research Ethics Committee (ID: 19196).

\section{Results}

Of the 3826 patients included in this study, 1047 (27\%) underwent ORP and 2779 (73\%) underwent RARP.

Differing baseline characteristics included that RARP patients were more likely to have low or intermediate NCCN disease risk (78.3\% vs. $72.0 \%, p<0.001)$ and reside in postcodes in the top quintile of the IRSAD $(43.9 \%$ vs. $34.3 \%, p<0.001$ ), compared to ORP patients (Table 1 ). RARP patients were also more likely to have surgery at a private institution $(85.3 \%$ vs. $60.6 \%, p<0.001)$ and have surgery at a metropolitan institution $(93.5 \%$ vs. $71.5 \%$, $p<0.001$ ), compared to ORP patients.

Table 2 shows the covariate balance in the propensity score matched cohort, compared to the unmatched cohort. After propensity score matching, no variables exceeded a standardised difference of $8 \%$.

\section{Urinary bother}

In the unmatched cohort, there was no statistically significant difference in the risk of reporting moderate/ big urinary bother in RARP compared to ORP patients (Rd $=-1.69 \%, P=0.125$ ) (Table 3). After adjusting for baseline characteristics in the propensity score matched cohort, there was also no significant difference in reporting moderate/big urinary bother $(\mathrm{Rd}=0.47 \%, P=0.707)$ (Table 3).

\section{Urinary incontinence}

In the unmatched cohort, there was no significant difference in urinary incontinence domain scores in RARP patients compared to ORP patients $($ Coeff $=1.27$, $P=0.195)$. In the propensity score matched cohort, there was also no significant difference in scores $($ Coeff $=-0.84, P=0.506)$.

\section{Pad usage}

In the unmatched cohort, there was no significant difference in the risk of wearing $\geq 1$ pad per day in RARP patients $(\mathrm{Rd}=-3.36 \%, P=0.074)$. In the propensity score matched cohort, there remained no significant difference in wearing $\geq 1$ pad per day $(R d=0.75 \%$, $P=0.771$ ). Sensitivity analysis with different cut-offs for binary variables can be seen in Additional file 1: Table S1, showing no difference between ORP and RARP in terms of pad usage, urinary bother and sexual bother.

\section{Sexual bother}

In the unmatched cohort, there was no significant difference in the risk of reporting moderate/big sexual bother in RARP compared to ORP patients $(\mathrm{Rd}=-0.33 \%$, $P=0.860$ ). In the propensity score matched cohort, the difference remained insignificant $(\mathrm{Rd}=-0.89 \%$, $P=0.731)$.

\section{Sexual domain}

In the unmatched sample, there were superior outcomes for men undergoing RARP, compared to those undergoing ORP, in sexual domain score $(30.15$ vs $23.48,<0.001$. In the propensity score matched cohort, this superiority persisted, but was less pronounced (29.57 vs. 25.92, respectively, $P=0.005$ ).

\section{Discussion}

This study used a large, registry-based cohort of patients and evaluated one-year urinary and sexual PROs. The unmatched cohort had different baseline characteristics, which we propose is due to a disparity in access to RARP. RARP patients were more likely to undergo surgery at metropolitan hospitals than ORP patients $(93.5 \%$ vs. $71.5 \%)$. RARP patients were also more likely to undergo surgery at private hospitals than ORP patients $(85.3 \%$ vs. $60.6 \%$ ). RARP patients had lower risk disease, which is potentially due to earlier screening.

Propensity score matching was used to decrease these and other baseline differences. After propensity score matching, no variables exceeded a standardised difference of $8 \%$. Many covariates had major reductions in standardised difference, including hospital location, hospital type and IRSAD category. The differences in four out of six PROs decreased once patient and surgeon 
Table 1 Baseline patient characteristics at diagnosis and surgery

\begin{tabular}{|c|c|c|c|}
\hline Factor & ORP & RARP & $P$ value \\
\hline N & $1047(27.4 \%)$ & $2779(72.6 \%)$ & \\
\hline \multicolumn{4}{|l|}{ Year of diagnosis } \\
\hline 2009-2012 & $3(0.3 \%)$ & $29(1.0 \%)$ & 0.05 \\
\hline 2013 & $31(3.0 \%)$ & $64(2.3 \%)$ & \\
\hline 2014 & $184(17.6 \%)$ & $507(18.2 \%)$ & \\
\hline 2015 & $226(21.6 \%)$ & $671(24.1 \%)$ & \\
\hline 2016 & $308(29.4 \%)$ & $750(27.0 \%)$ & \\
\hline 2017 & $279(26.6 \%)$ & $731(26.3 \%)$ & \\
\hline 2018 & $16(1.5 \%)$ & $27(1.0 \%)$ & \\
\hline \multicolumn{4}{|l|}{ Method of diagnosis } \\
\hline TRUS prostate biopsy & $516(49.3 \%)$ & $1160(41.7 \%)$ & $<0.001$ \\
\hline TURP & $16(1.5 \%)$ & $35(1.3 \%)$ & \\
\hline Transperineal prostate biopsy & $511(48.8 \%)$ & $1575(56.7 \%)$ & \\
\hline Other & $4(0.4 \%)$ & $9(0.3 \%)$ & \\
\hline \multicolumn{4}{|l|}{ PSA at diagnosis (ng/mL) } \\
\hline$<4.8$ & $212(20.2 \%)$ & $768(27.6 \%)$ & $<0.001$ \\
\hline $4.81-6.4$ & $238(22.7 \%)$ & $690(24.8 \%)$ & \\
\hline $6.41-9$ & $288(27.5 \%)$ & $652(23.5 \%)$ & \\
\hline$>9$ & $280(26.7 \%)$ & $593(21.3 \%)$ & \\
\hline Missing & $29(2.8 \%)$ & $76(2.7 \%)$ & \\
\hline \multicolumn{4}{|l|}{ ISUP Grade Group at diagnosis } \\
\hline 1 & $159(15.2 \%)$ & $417(15.0 \%)$ & $<0.001$ \\
\hline 2 & $412(39.4 \%)$ & $1301(46.8 \%)$ & \\
\hline 3 & $237(22.6 \%)$ & $578(20.8 \%)$ & \\
\hline 4 & $130(12.4 \%)$ & $272(9.8 \%)$ & \\
\hline 5 & $104(9.9 \%)$ & $181(6.5 \%)$ & \\
\hline Missing & $5(0.5 \%)$ & $30(1.1 \%)$ & \\
\hline \multicolumn{4}{|l|}{ NCCN risk category } \\
\hline Low risk & $89(8.5 \%)$ & $254(9.1 \%)$ & $<0.001$ \\
\hline Intermediate risk & $660(63.0 \%)$ & $1897(68.3 \%)$ & \\
\hline High risk & $256(24.5 \%)$ & $550(19.8 \%)$ & \\
\hline Metastatic & $36(3.4 \%)$ & $47(1.7 \%)$ & \\
\hline Missing & $6(0.6 \%)$ & $31(1.1 \%)$ & \\
\hline \multicolumn{4}{|l|}{ Diagnosing institution type } \\
\hline Public & $345(33.0 \%)$ & $415(14.9 \%)$ & $<0.001$ \\
\hline Private & $667(63.7 \%)$ & $2180(78.4 \%)$ & \\
\hline Missing & $35(3.3 \%)$ & $184(6.6 \%)$ & \\
\hline \multicolumn{4}{|l|}{ Diagnosing institution location } \\
\hline Metropolitan & $668(63.8 \%)$ & $2156(77.6 \%)$ & $<0.001$ \\
\hline Regional & $334(31.9 \%)$ & $426(15.3 \%)$ & \\
\hline Interstate/overseas & $8(0.8 \%)$ & $102(3.7 \%)$ & \\
\hline Missing & $37(3.5 \%)$ & 95 (3.4\%) & \\
\hline \multicolumn{4}{|l|}{ IRSAD category } \\
\hline 1-lowest & $123(11.7 \%)$ & $236(8.5 \%)$ & $<0.001$ \\
\hline 2 & $161(15.4 \%)$ & $285(10.3 \%)$ & \\
\hline 3 & $191(18.2 \%)$ & $362(13.0 \%)$ & \\
\hline 4 & $210(20.1 \%)$ & $673(24.2 \%)$ & \\
\hline 5-highest & $358(34.2 \%)$ & $1216(43.8 \%)$ & \\
\hline Missing & $4(0.4 \%)$ & $7(0.3 \%)$ & \\
\hline
\end{tabular}


Table 1 (continued)

\begin{tabular}{|c|c|c|c|}
\hline Factor & ORP & RARP & $P$ value \\
\hline Age at surgery, median (IQR) & $65.6(60.3,69.6)$ & $65.0(59.6,69.3)$ & 0.047 \\
\hline Years between diagnosis and surgery, median (IQR) & $0.2(0.1,0.3)$ & $0.2(0.1,0.3)$ & $<0.001$ \\
\hline Years between surgery and follow-up, median (IQR) & $1.0(1.0,1.1)$ & $1.0(1.0,1.1)$ & 0.86 \\
\hline \multicolumn{4}{|l|}{ PSA at surgery $(\mathrm{ng} / \mathrm{ml})$} \\
\hline$<4.8$ & $202(19.3 \%)$ & $774(27.9 \%)$ & \multirow[t]{5}{*}{$<0.001$} \\
\hline $4.81-6.4$ & $240(22.9 \%)$ & $661(23.8 \%)$ & \\
\hline $6.41-9$ & $286(27.3 \%)$ & $658(23.7 \%)$ & \\
\hline$>9$ & $298(28.5 \%)$ & $632(22.7 \%)$ & \\
\hline Missing & $21(2.0 \%)$ & $54(1.9 \%)$ & \\
\hline \multicolumn{4}{|l|}{ Pathological Tscore } \\
\hline $\mathrm{T} 2$ & $489(46.7 \%)$ & $1363(49.0 \%)$ & \multirow[t]{4}{*}{$<0.001$} \\
\hline T3 & $472(45.1 \%)$ & $1362(49.0 \%)$ & \\
\hline T4 & $4(0.4 \%)$ & $2(0.1 \%)$ & \\
\hline Missing & $82(7.8 \%)$ & $52(1.9 \%)$ & \\
\hline \multicolumn{4}{|l|}{ Surgery Gleason Score } \\
\hline 6 or less & $60(5.7 \%)$ & $98(3.5 \%)$ & \multirow[t]{6}{*}{$<0.001$} \\
\hline 7 & $771(73.6 \%)$ & $2301(82.8 \%)$ & \\
\hline 8 & $61(5.8 \%)$ & $114(4.1 \%)$ & \\
\hline 9 & $147(14.0 \%)$ & $241(8.7 \%)$ & \\
\hline 10 & $1(0.1 \%)$ & 0 & \\
\hline Missing & $7(0.7 \%)$ & $25(0.9 \%)$ & \\
\hline \multicolumn{4}{|l|}{ ISUP grade group at surgery } \\
\hline 1 & $136(13.0 \%)$ & $357(12.8 \%)$ & \multirow[t]{6}{*}{$<0.001$} \\
\hline 2 & $430(41.1 \%)$ & $1261(45.4 \%)$ & \\
\hline 3 & $306(29.2 \%)$ & $835(30.0 \%)$ & \\
\hline 4 & $80(7.6 \%)$ & $147(5.3 \%)$ & \\
\hline 5 & $91(8.7 \%)$ & $156(5.6 \%)$ & \\
\hline Missing & $4(0.4 \%)$ & $23(0.8 \%)$ & \\
\hline \multicolumn{4}{|l|}{ Surgical margins } \\
\hline Absent & $668(63.8 \%)$ & $2175(78.3 \%)$ & \multirow[t]{3}{*}{$<0.001$} \\
\hline Present & $356(34.0 \%)$ & $566(20.4 \%)$ & \\
\hline Missing & $23(2.2 \%)$ & $38(1.4 \%)$ & \\
\hline \multicolumn{4}{|l|}{ Surgical institution type } \\
\hline Public & 412 (39.4\%) & $402(14.5 \%)$ & \multirow[t]{3}{*}{$<0.001$} \\
\hline Private & $634(60.6 \%)$ & $2370(85.3 \%)$ & \\
\hline Missing & $1(0.1 \%)$ & $7(0.3 \%)$ & \\
\hline \multicolumn{4}{|l|}{ Surgical institution location } \\
\hline Metropolitan & 749 (71.5\%) & $2599(93.5 \%)$ & \multirow[t]{4}{*}{$<0.001$} \\
\hline Regional & $297(28.4 \%)$ & $173(6.2 \%)$ & \\
\hline Interstate/overseas & $0(0.0 \%)$ & $3(0.1 \%)$ & \\
\hline Missing & $1(0.1 \%)$ & $4(0.1 \%)$ & \\
\hline \multicolumn{4}{|l|}{ Surgeon year of specialisation } \\
\hline 1974-1990 & $139(13.3 \%)$ & $633(22.8 \%)$ & \multirow[t]{6}{*}{$<0.001$} \\
\hline $1991-2000$ & $430(41.1 \%)$ & $312(11.2 \%)$ & \\
\hline $2001-2006$ & $90(8.6 \%)$ & 799 (28.8\%) & \\
\hline 2007-2009 & $185(17.7 \%)$ & $434(15.6 \%)$ & \\
\hline 2010-2018 & $156(14.9 \%)$ & $568(20.4 \%)$ & \\
\hline Missing & 47 (4.5\%) & $33(1.2 \%)$ & \\
\hline Surgeon years since specialisation & & & \\
\hline
\end{tabular}


Table 1 (continued)

\begin{tabular}{lccc}
\hline Factor & ORP & RARP & P value \\
\hline $0-6$ & $167(16.0 \%)$ & $631(22.7 \%)$ & $630(22.7 \%)$ \\
$7-10$ & $169(16.1 \%)$ & $576(20.7 \%)$ \\
$11-16$ & $181(17.3 \%)$ & $321(11.6 \%)$ \\
$17-26$ & $339(32.4 \%)$ & $588(21.2 \%)$ \\
$27-44$ & $143(13.7 \%)$ & $33(1.2 \%)$
\end{tabular}

All continuous variables used a two-sample t-test. All categorical variables used a Pearson's chi-squared test. $P$ values exclude missing data

TRUS = Transrectal ultrasound, TUR $P=$ Transurethral resection of the prostate, PSA = Prostate-Specific Antigen, ISU $P=$ International Society of Urological Pathology, $\mathrm{NCCN}=$ National Comprehensive Cancer Network, IRSAD = Index of Relative Socioeconomic Advantage and Disadvantage

Table 2 Covariate balance table and standardised differences of variables included in the propensity score model

\begin{tabular}{|c|c|c|c|c|c|c|c|c|}
\hline \multirow[t]{2}{*}{ Factor } & \multicolumn{4}{|c|}{ Unmatched cohort } & \multicolumn{4}{|c|}{ Matched cohort } \\
\hline & ORP & RARP & $P$ value & $\begin{array}{l}\text { Standardised } \\
\text { difference }\end{array}$ & ORP & RARP & $P$ value & $\begin{array}{l}\text { Standardised } \\
\text { difference }\end{array}$ \\
\hline N & 971 & 2663 & & & 3634 & 3634 & & \\
\hline Age at surgery, mean (SD) & $64.9(6.8)$ & $64.3(7.1)$ & 0.028 & $-8.3 \%$ & $64.4(6.9)$ & $64.7(7.0)$ & 0.100 & $3.8 \%$ \\
\hline PSA at surgery, mean (SD) & $8.4(6.4)$ & $7.9(7.4)$ & 0.033 & $-8.3 \%$ & $7.9(6.1)$ & $8.0(8.1)$ & 0.510 & $1.5 \%$ \\
\hline $\begin{array}{l}\text { Surgeon years since spe- } \\
\text { cialisation, mean (SD) }\end{array}$ & $16.4(9.3)$ & $15.8(11.3)$ & 0.120 & $-6.0 \%$ & $16.5(8.4)$ & $16.4(11.5)$ & 0.540 & $-1.4 \%$ \\
\hline \multicolumn{9}{|l|}{ NCCN category } \\
\hline Low risk & $85(8.8 \%)$ & $240(9.0 \%)$ & $<0.001$ & & $325(8.9 \%)$ & $321(8.8 \%)$ & 0.630 & \\
\hline Intermediate risk & $617(63.5 \%)$ & $1853(69.6 \%)$ & & $12.8 \%$ & $2488(68.5 \%)$ & $2479(68.2 \%)$ & & $-0.5 \%$ \\
\hline High risk & $236(24.3 \%)$ & $526(19.8 \%)$ & & $-11.0 \%$ & $733(20.2 \%)$ & $760(20.9 \%)$ & & $1.8 \%$ \\
\hline Metastatic & $33(3.4 \%)$ & $44(1.7 \%)$ & & $-11.1 \%$ & $88(2.4 \%)$ & $74(2.0 \%)$ & & $-2.6 \%$ \\
\hline \multicolumn{9}{|l|}{ Hospital location } \\
\hline Metropolitan & $690(71.1 \%)$ & $2493(93.6 \%)$ & $<0.001$ & & $3161(87.0 \%)$ & $3184(87.6 \%)$ & 0.420 & \\
\hline Regional & $281(28.9 \%)$ & $170(6.4 \%)$ & & $-61.9 \%$ & $473(13.0 \%)$ & $450(12.4 \%)$ & & $-1.9 \%$ \\
\hline \multicolumn{9}{|l|}{ Hospital type } \\
\hline Public & 351 (36.1\%) & $366(13.7 \%)$ & $<0.001$ & & 715 (19.7\%) & $697(19.2 \%)$ & 0.590 & \\
\hline Private & 620 (63.9\%) & $2297(86.3 \%)$ & & $53.6 \%$ & 2919 (80.3\%) & $2937(80.8 \%)$ & & $1.3 \%$ \\
\hline \multicolumn{9}{|l|}{ IRSAD category } \\
\hline 1-lowest & 112 (11.5\%) & $224(8.4 \%)$ & $<0.001$ & & $274(7.5 \%)$ & 376 (10.3\%) & $<0.001$ & \\
\hline 2 & 150 (15.4\%) & $272(10.2 \%)$ & & $-15.7 \%$ & 395 (10.9\%) & 391 (10.8\%) & & $-0.4 \%$ \\
\hline 3 & 175 (18.0\%) & 345 (13.0\%) & & $-14.0 \%$ & 515 (14.2\%) & $528(14.5 \%)$ & & $1.0 \%$ \\
\hline 4 & 195 (20.1\%) & $652(24.5 \%)$ & & $10.6 \%$ & $937(25.8 \%)$ & 819 (22.5\%) & & $-7.6 \%$ \\
\hline 5-highest & 339 (34.9\%) & 1170 (43.9\%) & & $18.5 \%$ & $1513(41.6 \%)$ & $1520(41.8 \%)$ & & $0.4 \%$ \\
\hline \multicolumn{9}{|l|}{ Year of surgery } \\
\hline 2014 & $153(15.8 \%)$ & $417(15.7 \%)$ & 0.230 & & 467 (12.9\%) & $552(15.2 \%)$ & 0.040 & \\
\hline 2015 & 194 (20.0\%) & $598(22.5 \%)$ & & $6.1 \%$ & 759 (20.9\%) & 763 (21.0\%) & & $0.3 \%$ \\
\hline 2016 & 275 (28.3\%) & 709 (26.6\%) & & $-3.8 \%$ & 995 (27.4\%) & 960 (26.4\%) & & $-2.2 \%$ \\
\hline 2017 & 287 (29.6\%) & 807 (30.3\%) & & $1.6 \%$ & $1193(32.8 \%)$ & $1126(31.0 \%)$ & & $-4.0 \%$ \\
\hline 2018 & $62(6.4 \%)$ & $132(5.0 \%)$ & & $-6.2 \%$ & $220(6.1 \%)$ & 233 (6.4\%) & & $1.5 \%$ \\
\hline
\end{tabular}

Based on the propensity score matching model, the matching process created an observation for each patient if they were to receive the other treatment option and imputed the potential outcome of each observation. Therefore the matched cohort contained double the amount of observations $(n=7268)$ than the unmatched cohort $(n=3634)$. Before propensity score matching, standardised differences between ORP and RARP groups exceeded $10 \%$ for four of nine analysed covariates (NCCN category, hospital type, hospital location and IRSAD quintile). After propensity score matching, no variables exceeded a standardised difference of $8 \%$

All continuous variables used a two-sample t-test. All binary and categorical variables used the Pearson's chi-squared test. The matched cohort shows the distribution of covariates after matching for all patients that had answered the urinary bother item, as it had the highest number of responses out of all outcome variables 


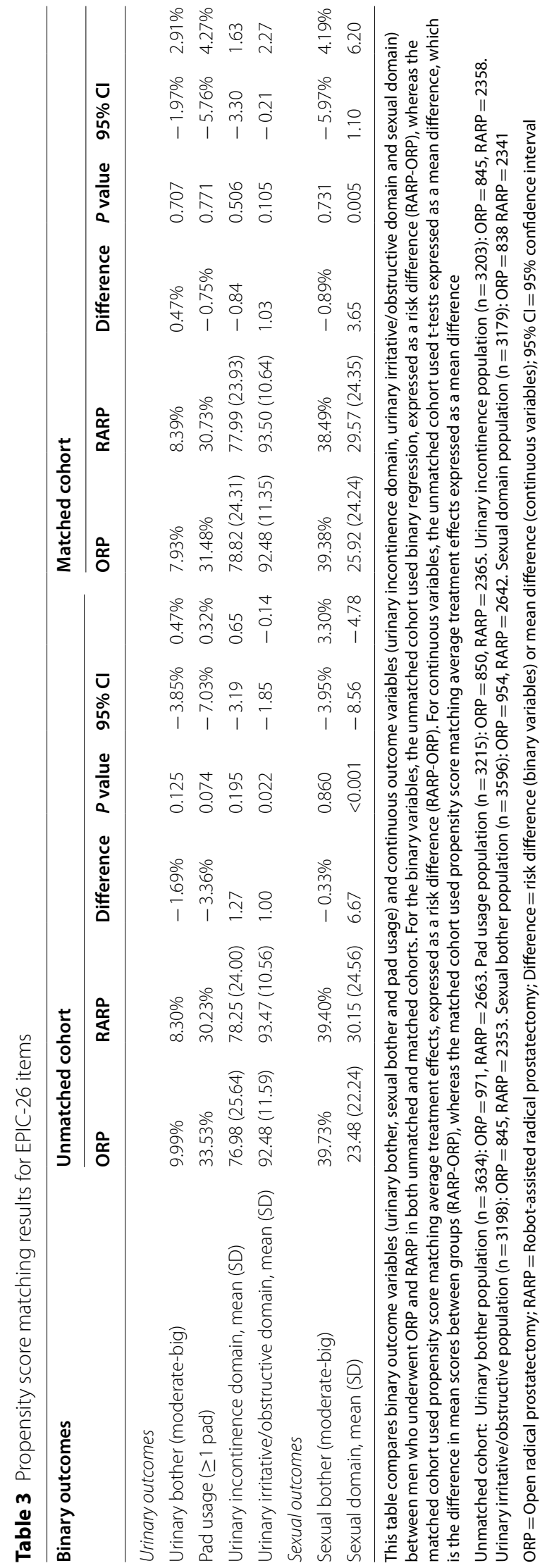


characteristics were more even amongst matched groups. This may be slightly due to an increased sample size from matching but is more likely due to a reduction in baseline differences.

Our results show that there is no statistically significant difference between ORP and RARP in reporting any urinary outcomes, including urinary bother, urinary incontinence, urinary irritative/obstructive and pad usage. Furthermore, there was no statistically significant difference between ORP and RARP in reporting sexual bother. Sexual domain scores were statistically superior in the RARP group. However, an absolute difference of less than 4 points on a 100 -point scale was deemed unlikely to be clinically significant [23]. In large datasets, including those collected by clinical registries, small differences in outcomes between treatment groups may be considered statistically significant even though they are not clinically meaningful [24].

\section{Urinary outcomes}

The largest RCT in the field reported no difference in urinary incontinence domain scores at 6 weeks, 12 weeks and 6-, 12- and 24-months [6,7]. However, they only used two surgeons with varying levels of experience and therefore their findings may be confounded by surgeon differences. Three studies have used propensity score matching to compare postoperative urinary outcomes between ORP and RARP $[18,25,26]$. Each reported no difference in urinary outcomes between ORP and RARP (Additional file 1: Table S1) [18, 25, 26].

The majority of studies in the field are observational and have retrospectively compared ORP and RARP. A previous study from the PCOR-Vic found no difference in 12-month urinary bother [18]. In contrast, Herlemann et al. concluded that men undergoing ORP were more likely to report superior EPIC-26 urinary incontinence domain scores and less bother than RARP patients within one year of surgery, in unadjusted analysis [27]. However, they also noted that the ORP group had significantly lower risk scores, Gleason grades and pT stages, which introduced selection bias. Results like these should be adjusted for baseline differences through techniques such as propensity score matching or regression analysis. Herlemann et al. hypothesised that the worse urinary outcomes for RARP patients in the first year may be influenced by increased expectations of RARP, especially in America [27].

One Swedish study found no difference between ORP and RARP in the number of pads worn per day for all daily cut-offs $(\geq 1, \geq 2, \geq 4$ and $\geq 6)$ [28]. We also found no difference between ORP and RARP patients in the unmatched and matched cohorts, with cut-offs at $\geq 1, \geq 2$ and $\geq 3$ pads per day (Additional file 1: Table S2).

\section{Sexual outcomes}

Our finding of marginal improvement in sexual domain scores at 12 months in the RARP group is comparable with two observational studies that used propensity score matching $[25,29]$. An Italian study by Antonelli et al. found a significant difference in 6-month unadjusted sexual function scores in favour of RARP by 12.41 points $(p<0.001)$, using the University of California Los Angeles Prostate Cancer Index (UCLA-PCI) questionnaire [25]. However, there was no difference at 12 months [25]. Antonelli et al. noted a clear trend towards centralisation of RARPs in Italy and therefore surgeon factors and volume-outcome relationships might explain the superior outcomes for RARP [25]. Similarly, RARP is centralised to metropolitan regions in Victoria, with greater access in private hospitals, which may skew views of its superiority.

Sooriakumaran et al. reported that patients in low or intermediaterisk groups recovered earlier from RARP, based on a single 5-scale penile stiffness question [29]. This was claimed to be due to RARP being a more flexible technique that has a greater capacity for nerve-sparing [29].

In a large American cohort, O'Neil et al. found a strong relationship between baseline function and function at later timepoints using common items from the UCLAPCI and EPIC questionnaires to form a modified sexual domain summary score [30]. Above a baseline sexual function score of $62 / 100$, RARP patients were found to have significantly improved sexual function at 12 months compared to ORP patients by a magnitude of 2.70 to 10.31 points [30]. This may indicate that RARP is able to preserve function in patients with higher preoperative function.

\section{Strengths and limitations}

Our study included data from 100 surgeons across metropolitan and regional Victoria. The use of propensity score matching allowed us to mimic a RCT by using matching in place of randomisation to create ORP and RARP patients who were alike on important baseline factors. It is important to understand the intricacies of each adjustment model and the variables selected in each model to assess the significance of outcomes. In contrast to other propensity score matching studies that used inverse probability treatment weighting $[18,25,26,29]$ we used 1:1 nearest neighbour matching, with replacement and no calipers. We included a number of known and novel factors in our propensity score model. Given the evidence that surgeon experience and skill are undervalued determinants of patient outcomes [31], our model included a novel variable, the number of years since the surgeon's specialisation. Furthermore, hospital location (metropolitan vs. regional) was included, which has 
not yet been examined as a predictor of quality of life. The effect of hospital type (private vs. public) will vary depending on each country's healthcare system. However, we included a hospital type variable as Victorian private sector patients have an increased likelihood of receiving RARP than public sector patients [32].

This study is not without limitations. First, the observational study design introduces the potential of unmeasured confounding and bias. The analysis was limited to outcome data collected at one timepoint and therefore no inferences could be made regarding time to recovery. Second, the absence of PRO data at baseline prevents adjusting for preoperative function. Participants of the PCOR-Vic are identified and contacted for consent a few months following diagnosis. Therefore collection of baseline data is not possible as many men will have had active management by the time they enter the registry. Furthermore, other variables were not collected by PCOR-Vic and therefore not included in the analysis, such as previous urological surgery and comorbidity data. Third, surgeries in the public sector are often performed by trainees under the supervision of nominal surgeons, and surgeon learning curve factors were not assessed. Therefore, our stratification of surgeon experience may not be truly representative. Fourth, the PCOR-Vic database had 75\% coverage of all RPs in Victoria in 2013 [33] and 89\% population coverage in 2019 [34], and captured data from patients at 61 sites (30/33 public and $31 / 41$ private). We believe the available data is representative of the Victorian population, however may not be applicable to other settings. Fifth, the PCOR-Vic database lacks standardised data collection for specimen handling and histological subtyping across all sites.

We suggest that future studies should capture surgeonspecific factors such as experience, skill and technique as well as ancillary services offered in the public and private sectors. Assessment of centralising services to high-volume centres is required as it may pool resources to better manage needs, yet may increase disparities in access to care [2]. Furthermore, the emergence of new surgical and radiological techniques, adjuvant treatments and medications, and demographic changes in patients and surgeons has the potential to change outcomes following RP and therefore requires ongoing assessment.

\section{Conclusion}

In this state-wide cohort of patients receiving ORP or RARP, there were no clinically significant differences in urinary PROs at one-year following RP. In terms of sexual function, there was slightly superior sexual domain scores for the RARP group, which was not deemed clinically significant.

\section{Abbreviations}

RARP: Robot-assisted radical prostatectomy; ORP: Open radical prostatectomy; PRO: Patient-reported outcome; RP: Radical prostatectomy; EPIC: Expanded Prostate cancer Index Composite questionnaire; RCT: Randomised controlled trial; IRSAD: Index of relative socioeconomic advantage and disadvantage.

\section{Supplementary Information}

The online version contains supplementary material available at https://doi. org/10.1186/s12894-022-00966-0.

Additional file 1. Figure S1. Balance plot of the probability of receiving RARP in both the ORP and RARP groups, in the unmatched and propensity score matched cohorts. Table S1. Summary of propensity score-matched studies comparing urinary and/or sexual outcomes following ORP vs. RARP. Table S2. Sensitivity analysis of binary outcomes dichotomised into different groups also found no significant differences between ORP and RARP in the unmatched or matched cohorts. Table S3. 12-month EPIC-26 urinary incontinence domain full responses. All items in the EPIC26 questionnaire refer to the last 4 weeks experienced by patients. This table compares individual items from the urinary incontinence domain between ORP and RARP. Table S4. 12-month EPIC-26 urinary irritative/ obstructive domain full responses. Table S5. 12-month EPIC-26 sexual domain full responses. Table S6. Other management/treatment options in cohort. Table S7. Subgroup analysis of the use of medications and aids for erectile function.

\section{Acknowledgements}

We acknowledge the funding support provided to the Prostate Cancer Outcomes Registry-Victoria by The Movember Foundation and the support of the registry by contributing clinicians, men diagnosed with prostate cancer and health institutions.

\section{Authors' contributions}

MR: Manuscript writing/editing, data analysis, protocol/project development. AF: Data analysis, manuscript writing/editing, protocol/project development. JLM: Manuscript editing, protocol/project development. ME: Manuscript editing, data collection, management and analysis. LD: Manuscript editing, surgeon expertise. DGM: Manuscript editing, surgeon expertise. SME: Protocol/project development, manuscript writing/editing. All authors read and approved the final manuscript.

Funding

Not applicable.

\section{Availability of data and materials}

Raw data were generated at the Prostate Cancer Outcomes Registry-Victoria. Derived data supporting the findings of this study are available from the corresponding author on request.

\section{Declarations}

\section{Ethics approval and consent to participate}

Informed consent was acquired from all participants of the study based on the recruitment strategy of the Prostate Cancer Outcomes Registry-Victoria. This study was performed in line with the principles of the Declaration of Helsinki. Approval was granted by the Monash University Human Research Ethics Committee (ID: 19196) (Approval date: 17.05.2019).

\section{Consent for publication}

Not applicable.

\section{Competing interests}

DGM reports personal fees from Janssen Pharma, personal fees from Astellas Pharma, personal fees from Bayer Pharma, personal fees from Ferring, personal fees from Astra Zeneca, personal fees from Ipsen, outside the submitted work. No other authors report any conflicts of interest. 


\section{Author details}

${ }^{1}$ School of Public Health and Preventive Medicine, Monash University, 533 St Kilda Road, Melbourne, VIC 3004, Australia. ${ }^{2}$ Radiation Oncology, Alfred Health, South Block Ground, 55 Commercial Road, Melbourne, VIC 3004, Australia. ${ }^{3}$ Department of Urology, Ballarat Health Services, Ballarat, Australia. ${ }^{4}$ St. John of God Hospital Ballarat, Ballarat, Australia. ${ }^{5}$ Epworth Prostate Centre, Epworth Healthcare, Richmond, VIC, Australia. ${ }^{6}$ Sir Peter MacCallum Department of Oncology, University of Melbourne, Parkville, VIC 3052, Australia. ${ }^{7}$ Victorian Cancer Registry, 615 St Kilda Rd, Melbourne, VIC 3004, Australia.

Received: 7 July 2021 Accepted: 10 January 2022

Published online: 07 February 2022

\section{References}

1. Evans MA, Millar JL, Earnest A, Frydenberg M, Davis ID, Murphy DG, et al. Active surveillance of men with low risk prostate cancer: evidence from the Prostate Cancer Outcomes Registry-Victoria. Med J Aust. 2018;208(10):443

2. Basto M, Sathianathen N, Marvelde L, Ryan S, Goad J, Lawrentschuk N, et al. Patterns-of-care and health economic analysis of robot-assisted radical prostatectomy in the Australian public health system. BJU Int. 2016:117(6):930-9.

3. Wang LL, Begashaw K, Evans M, Earnest A, Evans SM, Millar JL, et al. Patterns of care and outcomes for men diagnosed with prostate cancer in Victoria: an update. ANZ J Surg. 2018;88(10):1042.

4. Mottet N, van den Bergh RCN, Briers E, Van den Broeck T, Cumberbatch MG, De Santis M, et al. EAU-EANM-ESTRO-ESUR-SIOG guidelines on prostate cancer-2020 update. Part 1: screening, diagnosis, and local treatment with curative intent. Eur Urol. 2021;79(2):243-62.

5. Sanda MG, Cadeddu JA, Kirkby E, Chen RC, Crispino T, Fontanarosa J, et al. Clinically localized prostate cancer: AUA/ASTRO/SUO guideline. Part I: risk stratification, shared decision making, and care options. J Urol. 2018;199(3):683-90.

6. Coughlin GD, Yaxley JW, Chambers SK, Occhipinti S, Samaratunga H, Zajdlewicz L, et al. Robot-assisted laparoscopic prostatectomy versus open radical retropubic prostatectomy: 24-month outcomes from a randomised controlled study. Lancet Oncol. 2018;19(8):1051-60.

7. Yaxley JW, Coughlin GD, Chambers SK, Occhipinti S, Samaratunga $H$, Zajdlewicz L, et al. Robot-assisted laparoscopic prostatectomy versus open radical retropubic prostatectomy: early outcomes from a randomised controlled phase 3 study. The Lancet. 2016;388(10049):1057-66.

8. Finkelstein J, Eckersberger E, Sadri H, Taneja SS, Lepor H, Djavan B. Open versus laparoscopic versus robot-assisted laparoscopic prostatectomy: the European and US experience. Rev Urol. 2010;12(1):35-43.

9. Aggarwal A, Lewis D, Mason M, Purushotham A, Sullivan R, van der Meulen J. Effect of patient choice and hospital competition on service configuration and technology adoption within cancer surgery: a national, population-based study. Lancet Oncol. 2017;18(11):1445-53.

10. Frydenberg M, Murphy DG, Moon DA, Lawrentschuk N. Robotic assisted radical prostatectomy versus open retropubic radical prostatectomy: Where do we stand in 2015? Cancer Forum. 2015;39(3):173-7.

11. Schiffmann J, Larcher A, Sun M, Tian Z, Berdugo J, Leva I, et al. Differences in patient characteristics among men choosing open or robot-assisted radical prostatectomy in contemporary practice-analysis of surveillance, epidemiology, and end results database. Urol Int. 2017:98(1):48.

12. Chandrasekar T, Tilki R. Robotic-assisted vs open radical prostatectomy: an update to the never-ending debate. Transl Androl Urol. 2018;7(1):S120-3.

13. Gandaglia G, Bray F, Cooperberg MR, Karnes RJ, Leveridge MJ, Moretti $\mathrm{K}$, et al. Prostate cancer registries: current status and future directions. Eur Urol. 2016;69(6):998-1012.

14. Gershman B, Psutka SP, McGovern FJ, Dahl DM, Tabatabaei S, Gettman MT, et al. Patient-reported functional outcomes following open, laparoscopic, and robotic assisted radical prostatectomy performed by high-volume surgeons at high-volume hospitals. Eur Urol Focus. 2016:2(2):172-9.
15. Evans SM, Millar JL, Wood JM, Davis ID, Bolton D, Giles GG, et al. The Prostate Cancer Registry: monitoring patterns and quality of care for men diagnosed with prostate cancer. BJU Int. 2012;114(4 Part B):E158-66.

16. Szymanski KM, Wei JT, Dunn RL, Sanda MG. Development and validation of an abbreviated version of the expanded prostate cancer index composite instrument for measuring health-related quality of life among prostate cancer survivors. Urology. 2010;76(5):1245-50.

17. Australian Bureau of Statistics. Census of population and housing: socio-economic indexes for areas (SEIFA), Australia, 2016 Australia: ABS. 2016. Accessed 27 Aug 2019. https://www.abs.gov.au/ausstats/abs@. nsf/Lookup/by\%20Subject/2033.0.55.001 2016 Main\%20Features $\sim$ IRSAD 20.

18. Ong WL, Evans SM, Spelman T, Kearns PA, Murphy DG, Millar JL. Comparison of oncological and health-related quality of life outcomes between open and robot-assisted radical prostatectomy for localised prostate cancer-findings from the population-based Victorian Prostate Cancer Registry. BJU Int. 2016;118(4):563-9.

19. Wei JT, Dunn RL, Litwin MS, Sandler HM, Sanda MG. Development and validation of the expanded prostate cancer index composite (EPIC) for comprehensive assessment of health-related quality of life in men with prostate cancer. Urology. 2000;56(6):899-905.

20. Williamson EJ, Forbes A. Introduction to propensity scores. Respirology. 2014;19(5):625-35.

21. Austin PC. An introduction to propensity score methods for reducing the effects of confounding in observational studies. Multivar Behav Res. 2011;46(3):399-424.

22. Yao XI, Wang X, Speicher PJ, Hwang ES, Cheng P, Harpole DH, et al. Reporting and guidelines in propensity score analysis: a systematic review of cancer and cancer surgical studies. J Natl Cancer Inst. 2017;109(8):729.

23. Skolarus TA, Dunn RL, Sanda MG, Chang P, Greenfield TK, Litwin MS, et al. Minimally important difference for the expanded prostate cancer index composite short form. Urology. 2015;85(1):101-6.

24. Kaji AH, Rademaker AW, Hyslop T. Tips for analyzing large data sets from the JAMA surgery statistical editors. JAMA Surg. 2018;153(6):508-9.

25. Antonelli A, Palumbo C, Noale M, Porreca A, Maggi S, Simeone C, et al. Impact of surgical approach on patient-reported outcomes after radical prostatectomy: a propensity score-weighted analysis from a multicenter, prospective, observational study (the pros-IT CNR study). Urol Int. 2019;103(1):8-18.

26. Koo KC, Tuliao P, Yoon YE, Chung BH, Hong SJ, Yang SC, et al. Robotassisted radical prostatectomy in the Korean population: a 5-year propensity-score matched comparative analysis versus open radical prostatectomy. Int J Urol. 2014;21(8):781-5.

27. Herlemann A, Cowan JE, Carroll PR, Cooperberg MR. Community-based outcomes of open versus robot-assisted radical prostatectomy. Eur Urol. 2018;73(2):215-23.

28. Haglind E, Carlsson S, Stranne J, Wallerstedt A, Wilderäng U, Thorsteinsdottir $T$, et al. Urinary incontinence and erectile dysfunction after robotic versus open radical prostatectomy: a prospective, controlled. Nonrand Trial Eur Urol. 2015:68(2):216-25.

29. Sooriakumaran P, Pini G, Nyberg T, Derogar M, Carlsson S, Stranne J, et al. Erectile function and oncologic outcomes following open retropubic and robot-assisted radical prostatectomy: results from the LAParoscopic prostatectomy robot open trial. Eur Urol. 2018;73(4):618-27.

30. O'Neil B, Koyama T, Alvarez J, Conwill RM, Albertsen PC, Cooperberg $M R$, et al. The comparative harms of open and robotic prostatectomy in population based samples. J Urol. 2016;195(2):321-9.

31. Goldenberg MG, Goldenberg L, Grantcharov TP. Surgeon performance predicts early continence after robot-assisted radical prostatectomy. J Endourol. 2017;31(9):858-63.

32. Te Marvelde L, Milne RL, Hornby CJ, Chapman AB, Giles GG, Haines IE. Differences in treatment choices for localised prostate cancer diagnosed in private and public health services. Med J Aust. 2020;213(9):411-7.

33. Sampurno F, Earnest A, Kumari PB, Millar JL, Davis ID, Murphy DG, et al. Quality of care achievements of the prostate cancer outcomes registryVictoria. Med J Aust. 2016;204(8):319. 
34. Victorian Cancer Registry. Cancer in Victoria: statistics \& trends 2019

Cancer Council Victoria M, Victoria. Accessed 16 Dec 2020. 2020. https://

www.cancervic.org.au/research/vcr/publications.

\section{Publisher's Note}

Springer Nature remains neutral with regard to jurisdictional claims in published maps and institutional affiliations. 